\title{
ON RUNS OF RESIDUES ${ }^{1}$
}

\section{H. LEHMER AND EMMA LEHMER}

According to a theorem of Alfred Brauer [1] all sufficiently large primes have runs of $l$ consecutive integers that are $k$ th power residues, where $k$ and $l$ are arbitrarily given integers. In this paper we consider the question of the first appearance of such runs.

Let $p$ be a sufficiently large prime and let

$$
r=r(k, l, p)
$$

be the least positive integer such that

$$
r, \quad r+1, \quad r+2, \cdots, \quad r+l-1
$$

are all congruent modulo $p$ to $k$ th powers of integers $>0$. It is natural to ask, when $k$ and $l$ are given, how large is this minimum $r$ and are there primes $p$ for which $r$ is arbitrarily large? If we let

$$
\Lambda(k, l)=\limsup _{p \rightarrow \infty} r(k, l, p)
$$

then is $\Lambda$ infinite or finite, and if finite what is its value?

It is easy to see that

$$
\Lambda(2,2)=9
$$

so that every prime $p>5$ has a pair of consecutive quadratic residues which appears not later than the pair $(9,10)$. In fact if 10 is not a quadratic residue of $p$ then either 2 or 5 is, and so we have either $(1,2)$ or $(4,5)$ as a pair of consecutive residues.

By an elaboration of this reasoning $M$. Dunton has shown that

$$
\Lambda(3,2)=77,
$$

and more recently $\mathrm{W}$. H. Mills has shown that

$$
\Lambda(4,2)=1224 \text {. }
$$

Both of these proofs are as yet unpublished.

In contrast to these results we prove in this paper that

$$
\Lambda(2,3)=\infty \text {, }
$$

and

Presented to the Society, November 19, 1960 under the title On the distribution of consecutive quadratic and cubic residues; received by the editors December 5, 1960.

1 This paper is the result of unsupported research. 


$$
\Lambda(k, 4)=\infty,
$$

$k \leqq 1048909$.

In other words, by proper choice of $p$ the appearance of a run of 3 quadratic residues or of 4 higher residues can be postponed as long as desired.

Proof of (2). Let $N$ be a positive integer. Then it suffices to prove that there is a prime $p$ for which

$$
r(2,3, p)>N \text {. }
$$

Let

$$
q_{1}, q_{2}, \cdots, q_{t}
$$

be all the primes $\leqq N$.

By the quadratic reciprocity law, those primes which have a particular prime $q_{i}$ as a quadratic residue belong to a set of arithmetic progressions of common difference $4 q_{i}$. Those primes which have $q_{i}$ for a nonresidue likewise belong to another set of arithmetic progressions of difference $4 q_{i}$. If we combine the progressions of the first kind for every prime $q_{i} \equiv 1(\bmod 3)$ with those of the second kind for every prime $q_{i} \equiv 2(\bmod 3)$ and use Dirichlet's theorem on primes in arithmetic progressions we see that there exists a prime $p$ such that

$$
\left(\frac{q}{p}\right) \equiv q(\bmod 3) \quad(q \neq 3, q \leqq N) .
$$

Using the multiplicative property of Legendre's symbol we see that

$$
\left(\frac{m}{p}\right) \equiv m(\bmod 3) \quad(m \neq \equiv 0(\bmod 3), m \leqq N) .
$$

But among any three consecutive numbers $\leqq N$ there is one congruent to $-1(\bmod 3)$ and hence, by $(5)$, is a nonresidue of this prime $p$. Hence the first run of three consecutive quadratic residues lies beyond $N$. This proves (4) and (2).

PROOF OF (3). The following theorem enables one to prove that for $l \geqq 4, \Lambda(k, l)=\infty$ for all $k$ up to high limits. It is clear that for such a program one may confine $k$ to odd prime values and take $l=4$.

TheOREM A. Let $k$ and $p^{*}=k n+1$ be odd primes. Suppose further that 2 is not a kth power residue of $p^{*}$, and $p^{*}$ is small enough so that it has no run of 4 consecutive kth power residues. Then $\Lambda(k, 4)=\infty$.

For the proof we need the following lemma which is a special case of a theorem of Kummer [2]. 
Lemma. Let $k$ be an odd prime and $q_{1}, q_{2}, \cdots, q_{t}$ be any set of distinct primes different from $k$. Let $\gamma_{1}, \gamma_{2}, \cdots, \gamma_{t}$ be a set of kth roots of unity. Then there exist infinitely many primes $p \equiv 1(\bmod k)$ with corresponding kth power character $\chi$ modulo $p$ such that

$$
\chi\left(q_{i}\right)=\gamma_{i} \quad(i=1(1) t) .
$$

To prove the theorem let $N$ be an arbitrarily large integer and let $q_{1}, q_{2}, \cdots, q_{t}$ be the primes $\leqq N$ with the exception of the prime $p^{*}$. Choosing a nonprincipal character, let $\gamma_{i}$ be the $k$ th power character of $q_{i}$ modulo $p^{*}$. By the lemma there exist infinitely many primes $p \equiv 1(\bmod k)$ such that the $q$ 's have the same characters modulo $p$ as modulo $p^{*}$. By the multiplicative property of characters this will be true of all the integers $m \leqq N$ that are not divisible by $p^{*}$. Hence $p$ has no run of 4 consecutive residues $\leqq N$ unless one of these residues is a multiple of $p^{*}$. But two units on either side of this multiple of $p^{*}$ we find numbers congruent to $\pm 2\left(\bmod p^{*}\right)$ which are nonresidues of $p^{*}$ and hence of $p$. Hence there is also no run of 4 residues which includes a multiple of $p^{*} \leqq N$. This proves the theorem.

The fact that $\Lambda(3,4)=\infty$ follows from the theorem by setting $k=3$ and $p^{*}=7$. Similarly by taking $k=5$ and $p^{*}=11$ we have $\Lambda(5,4)=\infty$.

There is good reason to believe that $\Lambda(k, 4)=\infty$ for all $k$. To prove this it would suffice to prove for each prime $k$ the existence of a prime $p^{*}=k n+1$ satisfying the hypothesis of the theorem. If $n$ is not too large, then $p^{*}=k n+1$ will not have 4 consecutive $k$ th power residues. In fact $n$ is precisely the number of residues altogether. Trivially, if $n=2$ we have $\Lambda(k, 4)=\infty$ as with $k=3,5,11,23$, etc. With a little more effort we can prove

TheOREM B. If $n \leqq 12$ then $\Lambda(k, 4)=\infty$.

Proof. We may suppose that $k>5$. Let $p^{*}=k n+1$ be a prime not satisfying the hypothesis of Theorem A. This failure is not due to the fact that 2 is a residue of $p^{*}$. In fact if 2 were a residue, $p^{*}$ would divide $2^{n}-1$ by Euler's criterion. Since $n$ is even and $\leqq 12$ this restricts $p^{*}$ to the values

$$
3,5,7,11,13,17,31 \text {. }
$$

In each case the corresponding value of $k$ is $\leqq 5$. Hence 2 must be a nonresidue along with -2 and $(p \pm 1) / 2$. Hence we may suppose that $p^{*}$ has a run of 4 residues

$$
2<a, a+1, a+2, a+3<(p-1) / 2
$$


as well as the negatives of these modulo $p^{*}$. Besides these 8 residues there are the two residues congruent to $\pm(a+2) / a \not \equiv \pm 1$. These two are isolated since

$$
\frac{a+2}{a}-1=\frac{2}{a} \text { and } \frac{a+2}{a}+1=\frac{2}{a}(a+1)
$$

are obvious nonresidues. The reciprocals $\pm a /(a+2)$ are also isolated residues and they are new because

$$
\frac{a+2}{a} \equiv-\frac{a}{a+2}\left(\bmod p^{*}\right)
$$

implies

$$
a(a+2) \equiv-2\left(\bmod p^{*}\right)
$$

in which a product of two residues is congruent to a nonresidue. Including the residues \pm 1 we have accounted for at least 14 distinct $k$ th power residues of $p^{*}$. Hence $14 \leqq n \leqq 12$, a contradiction. Therefore $p^{*}$ must satisfy the hypothesis of Theorem $A$ and so $\Lambda(k, 4)=\infty$.

A more elaborate argument involving the factors of $3^{n}-1$ and the Fibonacci numbers yields a theorem in which the 12 in Theorem B is replaced by 36.

Let $p_{0}=k n_{0}+1$ be the least prime congruent to 1 modulo $k$. Primes $k$ for which $n_{0}(k) \geqq 38$ are relatively rare, only about $3 \%$ of all the primes $<50000$ by actual count. The least such prime is $k=1637$ with $n_{0}=38$, and the largest value for $n_{0}$ for primes less than 50000 is $n_{0}=80$ for $k=47303$. The values of $k<50000$ were calculated on the SWAC and were tested on the 7090 by John Selfridge for pairs of consecutive $k$ th power residues. It was discovered that in this range the only pairs are the trivial pairs $(\omega, \omega+1)$ and $\left(\omega^{2} \equiv p-\omega-1, \omega^{2}+1 \equiv p-\omega\right)$, which appear whenever $n_{0}$ is a multiple of six. Since such pairs cannot obviously combine to make a quadruplet they were eliminated from the next run, made entirely on the 7090 by John Selfridge, for $k \leqq 1048909$ in which no nontrivial pairs occurred. The largest value of $n_{0}=156$ occurred for $k=707467$. These numerical results for which we are very grateful enable us to state the following theorem, using Theorem A.

Theorem C. If $k \leqq 1048909$, then $\Lambda(k, 4)=\infty$.

More generally one can ask about the first appearance of $l$ consecutive numbers each with specified $k$ th power character modulo $p$ $=k n+1$, excluding of course the case already considered in which all 
the numbers are $k$ th power residues. This seemingly more difficult problem is unexpectedly simple. Regardless of $l$ the first appearance of such a set of consecutive numbers may be delayed indefinitely by proper choice of $p$. In fact if we set all the $\gamma$ 's in the lemma at 1 we can find primes $p$ having all the primes $\leqq N$ and hence all the numbers $\leqq N$ as $k$ th power residues. Hence if the specified characters contain as much as a single nonresidue the first appearance can be made to occur beyond $N$.

In a future paper, written jointly with W. H. Mills, we determine the finite numbers $\Lambda(5,2), \Lambda(6,2)$ and $\Lambda(3,3)$.

\section{REFERENCES}

1. Alfred Brauer, Über Sequenzen von Potenzresten, S.-B. Deutsch. Akad. Wiss. Berlin 1928, 9-16.

2. D. Hilbert, Die Theorie der algebraischen Zahlkörper, Jber. Deutsch. Math. Verein. 4 (1897), 426.

University of California, Berkeley 